\title{
Large Area Synthesis, Characterization, and Anisotropic Etching of Two Dimensional Tungsten Disulfide Films
}

\author{
Z. Mutlu ${ }^{\mathrm{a}}$, M. Ozkan ${ }^{\mathrm{b}}$ and C. S. Ozkan ${ }^{\mathrm{c}^{*}}$ \\ a. Materials Science and Engineering Program, University of California, Riverside, California, 92521, USA. \\ b. Department of Electrical and Computer Engineering, University of California, Riverside, California, 92521 , USA. \\ c. Department of Mechanical Engineering, University of California, Riverside, California, 92521, USA. \\ * Corresponding author: Cengiz S. Ozkan, cozkan@engr.ucr.edu
}

\begin{abstract}
Emergent properties of tungsten disulfide at the quantum confinement limit hold promise for electronic and optoelectronic applications. Here we report on the large area synthesis of atomically thin tungsten disulfide films with strong photoluminescence properties via sulfurization of the pre-deposited tungsten films. Detailed characterization of the pre-deposited tungsten films and tungsten disulfide films are performed using microscopy and spectroscopy methods. By directly heating tungsten disulfide films in air, we have shown that the films tend to be etched into a series of triangular shaped pits with the same orientations, revealing the anisotropic etching behavior of tungsten disulfide edges. Moreover, the dimensions of the triangular pits increase with the number of layers, suggesting a thickness dependent behavior of etching in tungsten disulfide films. This method offers a promising new avenue for engineering the edge structures of tungsten disulfide films.
\end{abstract}

Keywords: Crystal Growth, Etching, Raman Spectroscopy, XPS 


\section{Introduction}

Graphene-like two-dimensional (2D) semiconducting transition metal dichalcogenides (TMDs), especially tin disulfide $\left(\mathrm{WS}_{2}\right)$, with sizeable band gaps have showed vast potential for electronics [1] and optoelectronics [2] due to exotic physical and electronic properties, recently. $\mathrm{WS}_{2}$ is a layered semiconductor material with a direct band gap of $\sim 1.95 \mathrm{eV} \mathrm{[3]} \mathrm{in} \mathrm{monolayer}$ form. For electronics, the presence of a band gap allows field-effect transistors (FETs) to have high on/off ratios [4], and its ultrathin nature allows the channel length to be reduced relative to those fabricated with conventional semiconductors [5]. For optoelectronics, the direct band gap produces high photoconductivity [6], and strong photoluminescence (PL) [3]. To explore new fundamental properties and to further develop their electronic and optoelectronic applications, synthesis of large-area atomically thin $\mathrm{WS}_{2}$ layers with uniform properties by a facile and scalable method is an essential requirement.

Recent top-down approaches including mechanical exfoliation [7] and liquid exfoliation [8] to obtain high crystalline $\mathrm{WS}_{2}$ flakes have attracted considerable attention. However, lateral dimensions of the flakes synthesized by the exfoliation methods are limited to few microns, which limits their applications in large-scale electronics and optoelectronics.

In contrast, chemical vapor deposition (CVD) techniques have great potential in producing large-area $\mathrm{WS}_{2}$ over macroscopic sizes, which are ideal for integration with current CMOS platform. Typically, CVD growth of $\mathrm{WS}_{2}$ includes vapor phase reaction or deposition of gaseous metal and chalcogenide feed stocks $[9,10]$, and sulfurization or decomposition of the pre-deposited films $[11,12]$. 
Among the CVD techniques, sulfurization of the pre-deposited thin films is emerging as a quick and easy way to obtain large-area atomically thin $\mathrm{WS}_{2}$ films on insulating substrates. Recently, several studies have reported direct sulfurization of tungsten oxide $\left(\mathrm{WO}_{3}\right)$ films deposited either by thermal evaporation [12] or by atomic layer deposition (ALD) [11]. However, to the best of our knowledge, large-area growth of atomically thin $\mathrm{WS}_{2}$ films on silicon dioxide $\left(\mathrm{SiO}_{2}\right)$ substrates by sulfurization of the tungsten (W) films deposited by electronbeam (e-beam) deposition has not been reported yet.

In parallel with the recent advances in the synthesis of TMDs, numerous methods have been developed to enable their identification and characterization. PL spectroscopy is a powerful method of probing the electronic structure of TMDs [13]. Recent studies report [14] that molybdenum disulfide $\left(\mathrm{MoS}_{2}\right)$ shows laser-dependent PL spectra, where the PL intensity and position are affected by varying the duration and intensity of laser irradiation. To the best of our knowledge, there is no systematic study concerning the effect of laser power on PL spectra of $\mathrm{WS}_{2}$, which is essential to probing the PL characteristics of $\mathrm{WS}_{2}$.

Recently, there has been an increasing interest in the nanoscale control of edge structures of TMDs. Nanoscale control of edge structures offers new pathways toward fine tuning the electronic, optical, chemical, magnetic, and catalytic properties of TMDs [15-18]. One way to engineer edge structures of TMDs is heating them in an oxygen $\left(\mathrm{O}_{2}\right)$ environment $[\mathbf{1 9}, \mathbf{2 0}]$. For example, high density triangular pits with the molybdenum (Mo) or sulfur (S) terminated zigzag edges on the surface of $\mathrm{MoS}_{2}$ sheets have been obtained upon annealing them in air, which might arise from the anisotropic etching of the active $\mathrm{MoS}_{2}$ edge sites [19]. Such edge terminated $\mathrm{MoS}_{2}$ structures find applications in diverse catalytic reactions [21, 22]. As mentioned above, most 
studies so far have focused on the oxidative etching of $\mathrm{MoS}_{2}$. However, to the best of our knowledge, the etching behavior of $\mathrm{WS}_{2}$ films has yet to be experimentally studied.

In this study, we demonstrate the large-area synthesis of atomically thin $\mathrm{WS}_{2}$ films on $\mathrm{SiO}_{2}$ substrates. Briefly, the $\mathrm{W}$ films are deposited on $\mathrm{SiO}_{2}$ substrate by using e-beam deposition method. In the next step, the as-deposited $\mathrm{W}$ films are annealed at $500{ }^{\circ} \mathrm{C}$ and then sulfurized at $850{ }^{\circ} \mathrm{C}$ to obtain $\mathrm{WS}_{2}$ films. The fundamental morphologic, electronic, optical and chemical properties of the pre-deposited $\mathrm{W}$ films and $\mathrm{WS}_{2}$ films are investigated using optical microscopy, atomic force microscopy (AFM), scanning electron microscopy (SEM), X-ray photoemission spectroscopy (XPS), Raman spectroscopy and PL spectroscopy. We also systematically investigate the dependence of PL spectra of $\mathrm{WS}_{2}$ films on laser power. Furthermore, we introduce a simple method to etch $\mathrm{WS}_{2}$ films with well-oriented triangular-shaped pits by heating them in air.

\section{Experimental Section}

Materials synthesis: $\mathrm{WS}_{2}$ films were synthesized via three steps: i) deposition of the W films, ii) annealing of the W films, and iii) sulfurization of the annealed W films. First of all, the W films with a thickness of $\sim 15 \mathrm{~nm}$ were deposited on $\mathrm{SiO}_{2}$ substrates $\left(300 \mathrm{~nm}\right.$ thick $\mathrm{SiO}_{2}$ layer deposited thermally on Si wafer) by e-beam deposition method. The deposition of the films was carried out in a load-lock chamber (Temescal BJD 1800) at a base pressure of $\sim 10^{-6}$ Torr and deposition pressure of $\sim 10^{-5}$ Torr, with an average growth rate of $1 \AA / \mathrm{s}$. Next, the as-deposited W films were annealed at $500{ }^{\circ} \mathrm{C}$ in open air for $60 \mathrm{~min}$ in order to oxidize the films in a single-zone vacuum tube furnace with 2-inch diameter quartz tube (MTI GSL-1200X-50). The annealed W films were then put at the center of the heating zone, and $250 \mathrm{mg}$ of S powder $(99.5 \%$, Alfa Aesar) was put in a ceramic boat at the upstream where $\mathrm{S}$ starts evaporating when the 
temperature of the center of the tube is $525^{\circ} \mathrm{C}$. High-purity argon (Ar) gas with a constant flow of $100 \mathrm{sccm}$ was used as the carrier gas. After that, the furnace was pumped down to remove the air, and the pressure was stabilized at 250 Torr. Further, the center of heating zone was heated to $850{ }^{\circ} \mathrm{C}$ with a heating rate of $5{ }^{\circ} \mathrm{C} / \mathrm{min}$, and it was kept at that temperature for $10 \mathrm{~min}$. Finally it was naturally cooled down to room temperature. The etching of $\mathrm{WS}_{2}$ films was performed in the same furnace by simply heating them in air at $500{ }^{\circ} \mathrm{C}$ for $45 \mathrm{~min}$.

Characterization: The morphology of $\mathrm{WS}_{2}$ films was investigated using SEM (FIB NNS450). Raman spectra and PL spectra were collected using a Horiba system with a $532 \mathrm{~nm}$ laser. All spectra were calibrated using the $520.5 \mathrm{~cm}^{-1}$ line of a Si wafer. XPS characterization was carried out by using a Kratos AXIS ULTRADLD XPS system equipped with an Al Ka monochromated X-ray source and a $165-\mathrm{mm}$ mean radius electron energy hemispherical analyzer. The vacuum

pressure was kept below $3 \times 10^{-9}$ torr, and the neutralizer was applied during the data acquisition. AFM analysis was performed using a commercial AFM system (Multimode, Veeco) in tapping mode.

\section{Results and Discussion}

The morphology and thickness of the $\mathrm{W}$ films before and after annealing are determined by AFM analysis. The AFM height image (Figure 1a) of the as-deposited films reveals island type morphology with a height of about $15 \mathrm{~nm}$. The simultaneously recorded AFM phase contrast image (Figure 1b) reveals an additional contrast on the surface of islands, which may be attributed to the 'native oxide layer'. 

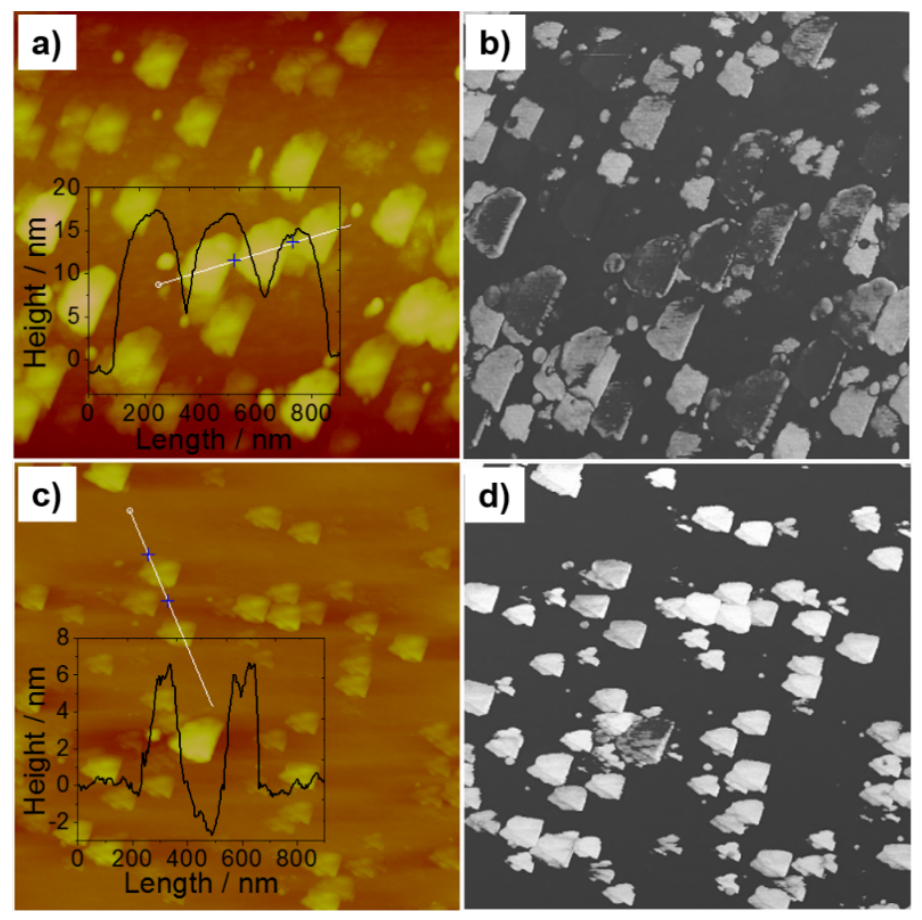

Figure 1 AFM height and corresponding phase images for the as-deposited (a, b) and annealed (c, d) $\mathrm{W}$ films on $\mathrm{SiO}_{2}$ substrates, respectively. The AFM height and phase contrast images are taken in an area of $2 \times 2 \mu \mathrm{m}^{2}$. The insets in (a) and (c) show the height profiles for the island morphologies.

The as-deposited $\mathrm{W}$ films are annealed in open air for $60 \mathrm{~min}$ at $500{ }^{\circ} \mathrm{C}$. Annealing is found to induce changes in the morphology and thickness of the films. The AFM height image (Figure 1c) of the annealed $\mathrm{W}$ films reveals roughly triangular island type morphologies with a height of about $6 \mathrm{~nm}$. While the density of the islands increases, the thickness of the islands decreases upon annealing. This could be explained as follows: It is know that when Tammann temperature $\left(\sim 599^{\circ} \mathrm{C}\right.$ for $\left.\mathrm{WO}_{3}\right)[\mathbf{2 3}]$ is achieved, the evaporation of metal oxide thin films can be initiated even though the processing temperature is lower than their melting point $\left(\sim 1473{ }^{\circ} \mathrm{C}\right.$ for $\mathrm{WO}_{3}$ ). It is possible that surface oxidation of the as-deposited $\mathrm{W}$ films may occur during deposition. Thus, the as-deposited $\mathrm{W}$ films with the native oxide layer might become thinner 
possibly due to the partial evaporation of the metal oxide layer upon annealing. The AFM phase contrast image (Figure 1d) reveals layered-type island morphologies with one single phase contrast, which can indicate a completed oxidation.

The as-deposited and annealed W films are investigated by XPS analysis. The highresolution XPS spectra (Figure 2a) of the as-deposited films reveal four major peaks at 37.99 $\mathrm{eV}, 35.87 \mathrm{eV}, 33.38 \mathrm{eV}$ and $31.2 \mathrm{eV}$. The typical doublet $\mathrm{W} 4 \mathrm{f}$ peaks of $\mathrm{W}$ are clearly visible in the spectra, which are at $33.38 \mathrm{eV}$ and $31.2 \mathrm{eV}$ [9]. The two upper binding energy peaks at 37.99 $\mathrm{eV}, 35.87 \mathrm{eV}$ can be attributed to $\mathrm{WO}_{3}$ [24], confirming the existence of $\mathrm{WO}_{3}$ in the as-deposited films. It is also found that there is a shoulder at upper energy side of each $\mathrm{W}$ peak, and the shoulders are related to $\mathrm{WO}_{2}$ and $\mathrm{WO}_{\mathrm{x}}$ [24]. We can conclude that $\mathrm{W}, \mathrm{WO}_{3}$ and substoichiometry tungsten-oxides exist in the as-deposited film. The high-resolution XPS spectra (Figure 2b) of the annealed films reveal only two $\mathrm{W} 4 \mathrm{f}$ peaks at $37.97 \mathrm{eV}$ and $35.87 \mathrm{eV}$, which are assigned to $\mathrm{WO}_{3}$. After annealing, the metallic $\mathrm{W}$ peaks and the shoulder peaks related to $\mathrm{WO}_{2}$ or $\mathrm{W}_{\mathrm{x}}$ completely disappear. This evidence strongly supports the stoichiometry phase evolution of the as-deposited films.

The XPS data of $\mathrm{WS}_{2}$ films are also captured to analyze surface composition of the $\mathrm{WS}_{2}$ films. The two peaks of each core level appear due to spin orbital splitting of W $4 \mathrm{f}$ (Figure 2c) and S 2p (Figure 2d) core levels. The peaks at $38.89 \mathrm{eV}, 35.40 \mathrm{eV}$, and $33.2 \mathrm{eV}$ are ascribed to $\mathrm{W} 5 \mathrm{p}_{3 / 2}, \mathrm{~W} 4 \mathrm{f}_{5 / 2}$, and $\mathrm{W} 4 \mathrm{f}_{7 / 2}$ orbitals, respectively. The binding energies for the $\mathrm{S} 2 \mathrm{p}_{1 / 2}$ and $\mathrm{S}$ $2 \mathrm{p}_{3 / 2}$ are located at $164.0 \mathrm{eV}$ and $162.82 \mathrm{eV}$, respectively. The binding energies of the $\mathrm{W}$ and $\mathrm{S}$ elements are consistent with the W (+4) and S (-2) oxidation states in $\mathrm{WS}_{2}$, further confirming the formation of a pure $\mathrm{WS}_{2}$ phase [25]. 

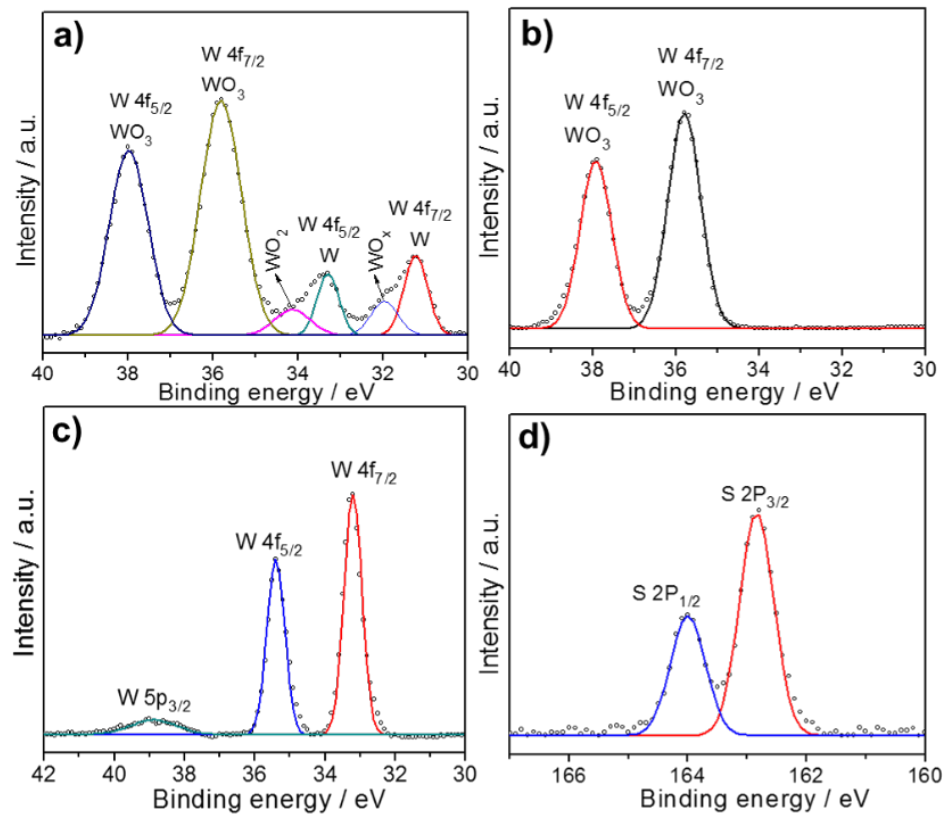

Figure 2 High resolution XPS spectra of W 4f core levels of the as-deposited (a) and annealed (b) W films. High resolution XPS spectra of W $4 \mathrm{f}$ (c) and S $2 p$ (d) core levels of $\mathrm{WS}_{2}$ films.

To study the effect of annealing on the growth of $\mathrm{WS}_{2}$ films, we have synthesized $\mathrm{WS}_{2}$ films by sulfurizing the as-deposited and annealed $\mathrm{W}$ films. It is observed that sulfurization of the as-deposited $\mathrm{W}$ films leads to thick $\mathrm{WS}_{2}$ films with poor coverage (Figure 3a, b). However, thinner $\mathrm{WS}_{2}$ films with high coverage are obtained by sulfurization of the annealed $\mathrm{W}$ films (Figure 3c, d). For clarification, monolayer, few-layers and thick regions of $\mathrm{WS}_{2}$ films and the substrate are indicated by arrows on the high magnification SEM images (Figure $\mathbf{3 b}$, d), as confirmed by Raman and PL spectroscopy. We can conclude that the $\mathrm{WS}_{2}$ growth is substantially improved by annealing the as-deposited $\mathrm{W}$ films before sulfurization.

The growth mechanism of $\mathrm{WS}_{2}$ films can be explained as follows. The reaction of $\mathrm{S}$ vapor with $\mathrm{WO}_{3}$ islands result in $\mathrm{WS}_{2}$ nuclei. This reaction includes a transition to $\mathrm{WO}_{(3-\mathrm{x})}$ species along with the subsequent formation of oxisulfides. As the reaction proceeds, a complete 
conversion to $\mathrm{WS}_{2}$ occurs, and thinner $\mathrm{WS}_{2}$ layers expand from the edges of $\mathrm{WS}_{2}$ nuclei, in a similar manner as in $\mathrm{MoS}_{2}[\mathbf{2 6}]$.

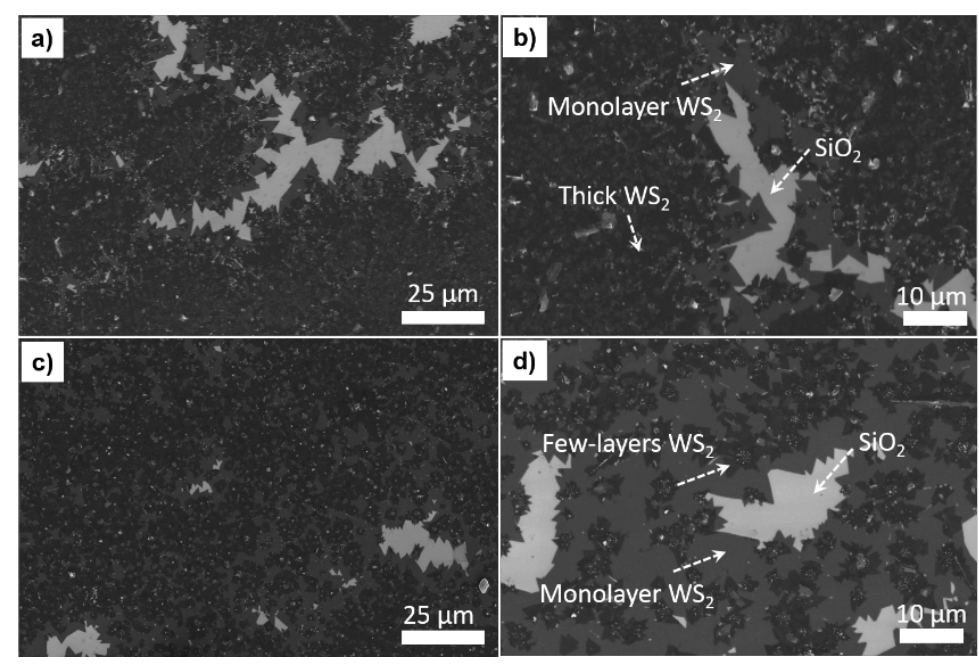

Figure 3 Low and high magnification SEM images of $\mathrm{WS}_{2}$ films obtained by sulfurizing the asdeposited (a, b) and annealed (c, d) W films, respectively.

We have taken Raman and PL spectra from monolayer and few-layers regions of a $\mathrm{WS}_{2}$ film as indicated by arrows on the optical microcopy image (Figure 4a) of the film. The Raman spectra (Figure $4 \mathbf{b}$ ) of monolayer region of the $\mathrm{WS}_{2}$ film demonstrate the characteristic in-plane vibrational $\left(\mathrm{E}_{2 \mathrm{~g}}^{1}\right)$ mode and the out-of-plane vibrational $\left(\mathrm{A}_{1 \mathrm{~g}}\right)$ mode at $\sim 350 \mathrm{~cm}^{-1}$ and $\sim 416 \mathrm{~cm}^{-}$ ${ }^{1}$, respectively $[27,28]$. The frequency difference between the two modes is found to be $\sim 66 \mathrm{~cm}^{-}$ 1. These results are consistent with what has been reported for $\mathrm{WS}_{2}$ in the previous studies [29]. The PL spectra (Figure 4c) of monolayer and few-layers regions of the $\mathrm{WS}_{2}$ film are compared. For monolayer $\mathrm{WS}_{2}$, the PL peak has a maxima at $1.96 \mathrm{eV}$, which falls in the range of the reported PL peak positions for monolayer $\mathrm{WS}_{2}\left[\right.$ [7, 29]. The PL peak of monolayer $\mathrm{WS}_{2}$ is also higher and sharper than that of the few-layers $\mathrm{WS}_{2}$, which indicates the presence of a direct band gap in monolayer $\mathrm{WS}_{2}\left[\mathbf{3 0 ]}\right.$. For few-layers $\mathrm{WS}_{2}$, the PL peak shifts toward lower photon energy 
$(\sim 1.93 \mathrm{eV})$, and its intensity decreases dramatically, revealing the thickness dependent PL characteristics of $\mathrm{WS}_{2}$.
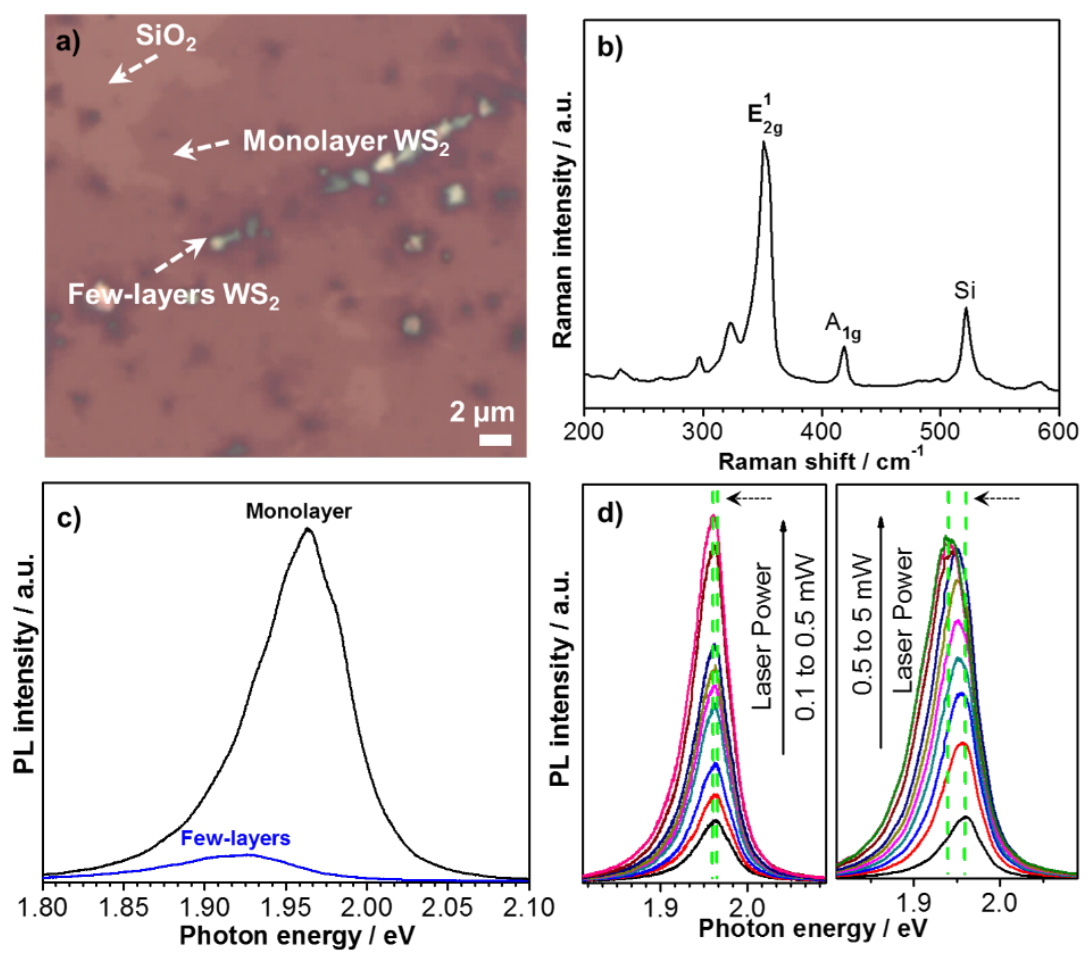

Figure 4 a) Optical microscopy image of a $\mathrm{WS}_{2}$ film. Monolayer and few-layers regions of the $\mathrm{WS}_{2}$ film and the substrate are indicated by arrows b) Raman spectra of monolayer region of the $\mathrm{WS}_{2}$ film. c) PL spectra of monolayer and few-layers regions of the $\mathrm{WS}_{2}$ film. d) Laser power dependent PL spectra of monolayer region of the $\mathrm{WS}_{2}$ film.

We have also investigated the effect of laser power on PL spectra of monolayer region of the $\mathrm{WS}_{2}$ film with increasing laser power ranging from $0.1 \mathrm{~mW}$ to $0.5 \mathrm{~mW}$ with a step of 0.05 $\mathrm{mW}$ and $0.5 \mathrm{~mW}$ to $5 \mathrm{~mW}$ with a step of $0.5 \mathrm{~mW}$ (Figure 4d), respectively. It is observed that the PL intensity increases with increasing laser power, consisted with the results reported for $\mathrm{MoS}_{2}$ [14]. Moreover, the PL peak shifts toward lower photon energy as laser power increases. The shift in the PL peak can be attributed to the defect related optical transition [31]. Thus, we 
conclude that laser power must be carefully controlled in the applications of PL spectroscopy in order to draw reliable conclusions about the PL characteristics of TMDs.

Furthermore, by directly heating a $\mathrm{WS}_{2}$ film in air at $500{ }^{\circ} \mathrm{C}$, we show that the $\mathrm{WS}_{2}$ film is tend to be etched with a series of the triangular-shaped pits with the same orientations (Figure 5). The triangular shape of the pits indicates that the $\mathrm{WS}_{2}$ film is etched preferentially along a specific crystallographic direction that is parallel to the edges of the pits with a preferential edge termination (W-edge or S-edge) [19]. The etching is likely to be initiated at the intrinsic structural defects in the $\mathrm{WS}_{2}$ film [19]. We also show that the size of the pits are greatly affected by the $\mathrm{WS}_{2}$ film thickness, where the sizes of the pits increases with the thickness. The sizes of the triangular pits on a multilayers region (with a thickness of about $10.2 \mathrm{~nm}$ ) of the $\mathrm{WS}_{2}$ film (Figure 5a) is found to be larger than these of the pits on a few-layers region (with a thickness of about $3.2 \mathrm{~nm}$ ) of the film (Figure 5b). This suggests the thickness dependent behavior of the etching on the $\mathrm{WS}_{2}$ film [32]. These results are similar to previous reports on the etching of $\mathrm{MoS}_{2}[19,32,33]$.

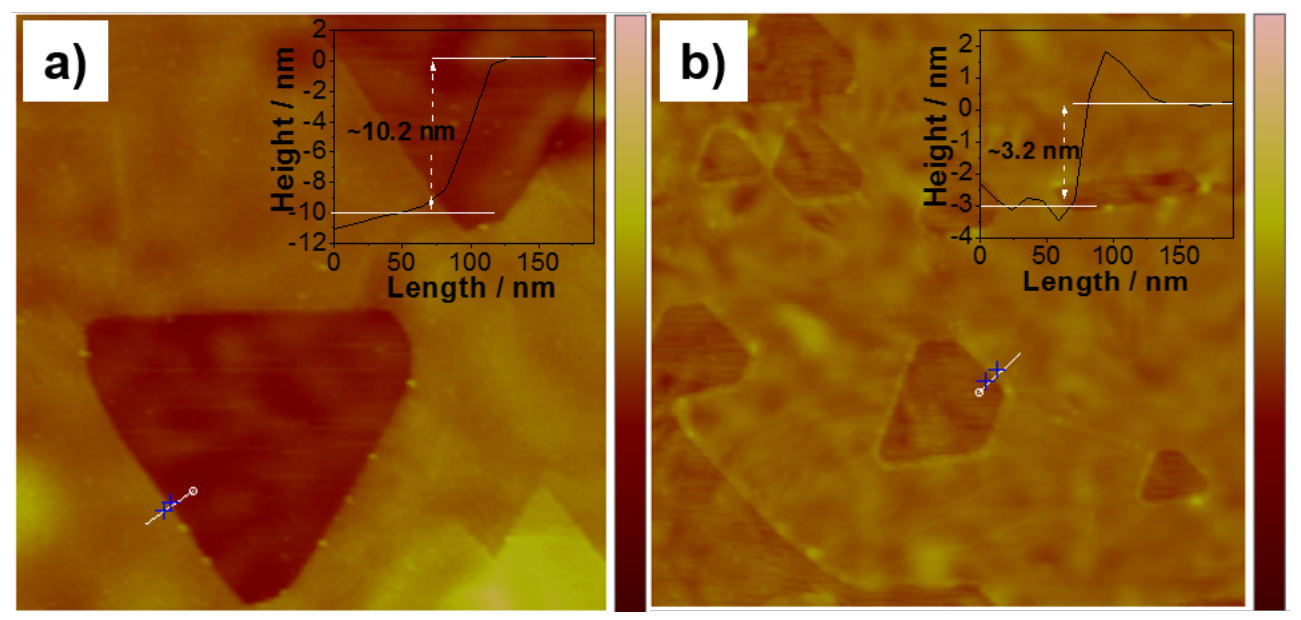


Figure 5 AFM height images showing the formation of the triangular pits on multilayers (a) and few-layers (b) regions of a $\mathrm{WS}_{2}$ film. The AFM images are taken in an area of $2 \times 2 \mu \mathrm{m}^{2}$. The insets in (a) and (b) show the height profiles for the triangular pits.

\section{Conclusion}

In summary, we report a facile method for the large-scale synthesis of atomically thin $\mathrm{WS}_{2}$ films with strong PL properties via sulfurization of the pre-deposited $\mathrm{W}$ films. Thermal annealing of the $\mathrm{W}$ films before sulfurization is found to substantially improve the quality of $\mathrm{WS}_{2}$ films in terms of thickness and uniformity. We also find that $\mathrm{WS}_{2}$ films show laser power dependent PL characteristics, where the PL peak shifts toward lower photon energy, and its intensity increases with laser power. Furthermore, we introduce a simple method to etch $\mathrm{WS}_{2}$ films with well-oriented triangular pits with a preferential edge termination. The edge sizes of the pits increase with the thickness of $\mathrm{WS}_{2}$ films. This etching method enables successful formation and modification of the edge structures of $\mathrm{WS}_{2}$ films with potentially tunable electronic, optical and magnetic properties, which promise various applications ranging from catalysis to energy harvesting.

\section{Acknowledgements}

The authors would like to thank Dr. Darshana Wickramaratne (from UCSB) for valuable feedback. Financial support for this work was provided by the STARnet center C-SPIN (Center for Spintronic Materials, Interfaces, and Novel Architectures) through the Semiconductor Research Corporation sponsored by MARCO and DARPA. XPS data were acquired with equipment funded by the NSF, Grant No. DMR-0958796. 


\section{References}

[1] T. Georgiou, R. Jalil, B. D. Belle, L. Britnell, R. V. Gorbachev, S. V. Morozov, Y. -J. Kim, A. Gholinia, S. J. Haigh, O. Makarovsky, L. Eaves, L. A. Ponomarenko, A. K. Geim, K. S. Novoselov and A. Mishchenko, Nat. Nanotechnol. 8, 100 (2013).

[2] N. Huo, J. Kang, Z. Wei, S. -S. Li, J. Li, and S. -H. Wei, Adv. Funct. Mater. 24, 7025 (2014).

[3] H. R. Gutiérrez , N. Perea-López, A. L. Elías, A. Berkdemir, B. Wang, R. Lv, F. LópezUrías, V. H. Crespi, H. Terrones and M. Terrones, Nano Lett. 13, 3447 (2013).

[4] W. S. Hwang, M. Remskar, R. Yan, V. Protasenko, K. Tahy, S. D. Chae, P. Zhao, A. Konar, H. G. Xing, A. Seabaugh and D. Jena, Appl. Phys. Lett. 10, 013107 (2012).

[5] D. Ovchinnikov, A. Allain, Y. -S. Huang, D. Dumcenco, and A. Kis, ACS Nano 8, 8174 (2014).

[6] S. Hwan Lee, D. Lee, W. S. Hwang, E. Hwang, D. Jena, and W. J. Yoo, Appl. Phys. Lett. 104, 193113 (2014).

[7] W. Zhao, Z. Ghorannevis, L. Chu, M. Toh, C. Kloc, P.-H. Tan and Goki Eda, ACS Nano 7, 791 (2013).

[8] D. Voiry, H. Yamaguchi, J. Li, R. Silva, D. C. B. Alves, T. Fujita, M. Chen, T. Asefa, V. B. Shenoy, G. Eda, and M. Chhowalla, Nat. Mater. 12, 850 (2013).

[9] Y. Zhang, Y. Zhang, Q. Ji, J. Ju, H. Yuan, J. Shi, T. Gao, D. Ma, M. Liu, Y. Chen, X. Song, H. Hwang, Y. Cui and Zhongfan Liu, ACS Nano 7, 8963 (2013).

[10] C. Cong, J. Shang, X. Wu, B. Cao, N. Peimyoo, C. Qiu, L. Sun and T. Yu, Adv. Optical Mater. 2, 131 (2014). 
[11] J. G. Song, J. Park, W. Lee, T. Choi, H. Jung, C. W. Lee, S. H. Hwang, J. M. Myoung, J. H. Jung, S. H. Kim, C. Lansalot-Matras, and H. Kim, ACS Nano 7, 11333 (2013).

[12] A. L. Elías, N. Perea-López, A. Castro-Beltrán, A. Berkdemir, R. Lv, S. Feng, A. D. Long, T. Hayashi, Y. A. Kim, M. Endo, H. R. Gutiérrez, N. R. Pradhan, L. Balicas, T. E. Mallouk, F. López-Urías, H. Terrones and M. Terrones, ACS Nano 7, 5235 (2013).

[13] K. F. Mak, C. Lee, J. Hone, J. Shan, and T. F. Heinz, Phys. Rev. Lett. 105, 2 (2010).

[14] V. Senthilkumar, L. C. Tam, Y. S. Kim, Y. Sim, M. -J. Seong and J. I. Jang, Nano Res. 7, 1759 (2014).

[15] F. López-Urías, A. L. Elías, N. Perea-López, H. R Gutiérrez, M. Terrones, and H. Terrones, 2D Materials 2, 015002 (2015).

[16] S. Helveg, J. V. Lauritsen, E. Lægsgaard, I. Stensgaard, J. K. Nørskov, B. S. Clausen, H. Topsøe and F. Besenbacher, Phys. Rev. Lett. 84, 951 (2000).

[17] H. Zhang, X. -B. Li, and L. -M. Liu, J. Appl. Phys. 144, 093710 (2013).

[18] P. Murugan, V. Kumar, Y. Kawazoe and N. Ota, Phys. Rev. A 71, 063203 (2005).

[19] M. Yamamoto, T. L. Einstein, M. S. Fuhrer, and W. G. Cullen, J. Phys. Chem. C 117, 5643 (2013).

[20] R. Ionescu, A. George,I. Ruiz, Z. Favors, Z. Mutlu, C. Liu, K. Ahmed, R. Wu, J. S. Jeong, L. Zavala, K. A. Mkhoyan, M. Ozkan and C. S. Ozkan, Chem. Commun. 50, 11226 (2014).

[21] T. F. Jaramillo, K. P. Jørgensen, J. Bonde, J. H. Nielsen, S. Horch, and I. Chorkendorff, Science 317, 100 (2007). 
[22] J. Kibsgaard, Z. Chen, B. N. Reinecke, and T. F. Jaramillo, Nat. Mater. 11, 963 (2012).

[23] D. P. Debecker, R. Delaigle, K. Bouchmella, P. Eloy, E. M. Gaigneaux, and P. H. Mutin, Catal. Today 157, 125 (2010).

[24] X. P. Wang, B. Q. Yang, H. X. Zhang, and P. X. Feng, Nanoscale Res. Lett. 2, 405 (2007).

[25] W. Yang, J. Wang, C. Si, Z. Peng, J. Frenzel, G. Eggeler, and Z. Zhang, J. Mater. Chem. A 3, $17811(2015)$.

[26] R. Ionescu, W. Wang, Y. Chai, Z. Mutlu, I. Ruiz, Z. Favors, D. Wickramaratne, M. Neupane, L. Zavala, M. Ozkan and C. S. Ozkan, Trans. Nanotechnol. 13, 749 (2014).

[27] T. Sekine, T. Nakashizu, K. Toyoda, K. Uchinokura, and E. Matsuura, Solid State Commun. $35,371(\mathbf{1 9 8 0})$.

[28] C. Sourisseau, F. Cruege, M. Fouassier, and M. Alba, Chem. Phys. 150, 281 (1991).

[29] Y. Rong, Y. Fan, A. Leen Koh, A. W. Robertson, K. He, S. Wang, H. Tan, R. Sinclair, and J. H. Warner, Nanoscale 6, 12096 (2014).

[30] D. Wickramaratne, F. Zahid, and R. K. Lake, J. Chem. Phys. 140, 124710 (2014).

[31] P. K. Chow, R. B. J. -Gedrim, J. Gao, T. M. Lu, B. Yu, H. Terrones and N. Koratkar, ACS Nano 9, 1520 (2015).

[32] H. Zhou, F. Yu, Y. Liu, X. Zou, C. Cong, C. Qiu, and T. Yu, Nano Res. 6, 703 (2013).

[33] Jumiati Wu, Hai Li, Zongyou Yin, Hong Li, Juqing Liu, Xiehong Cao, Qing Zhang, Hua Zhang, Small 9, 3314 (2013). 